\title{
LEAD OXIDE GLASS TUBING CONVERTERS FOR GAMMA DETECTION IN MWPC
}

G.K. Lum, M. I. Green, V. Perez-Mendez and K.C. Tam

Lawrence Berkeley Laboratory, Berkeley and

Radiology Department, U.C. San Francisco

\section{Abstract}

Pb0 glass converters for the detection of gamma rays with MWPC have been investigated. The converters were made by fusing glass tubes stacked to form a close-packed array and were treated in a $\mathrm{H}_{2}$ reduction process to provide a suitable electric drift field. Electron conversion efficiency, drift velocity, and spatial resolution of these arrays were investigated. Using $511 \mathrm{keV}$ gamma rays, an electron conversion efficiency of $8 \%$ was established for the converter of $80 \% \mathrm{PbO}$ by weight ( $1.4 \mathrm{~mm}$ inner diameter, $0.1 \mathrm{~mm}$ wall thickness and $2 \mathrm{~cm}$ thick).

\section{Introduction}

In using MWPC as detectors for gamma rays it is necessary to provide a suitable region of the chamber in which gamma rays can yield conversion electrons. At low energies, $E_{\gamma}<100 \mathrm{keV}$, xenon gas up to a few atmospheres of pressure can be used. For high energy gamma rays, $E_{\gamma}>10 \mathrm{MeV}$, high Z-element solid converters can be used since the shower process yields primary electrons which have enough energy to escape into the gas region where they are detected by the ionization electrons they produce.

High efficiency converters for intermediate energy gamma rays with energies between $100 \mathrm{KeV}$ and a few MeV require special converters of high $Z$ materials. These converters have a large surface-to-volume ratio such that secondary electrons can be drifted out by suitable electric fields into the sensitive region of a MWPC.

Figure 1 shows schematically the layout of such converters coupled to a conventional three wire plane MWPC. As indicated in the figure, the position accuracy for locating gamma rays depends both on the hole or slot size as well as on the thickness of the converter if we assume that the gamma ray flux incident on the chamber has a wide angular spread.

High $\mathrm{Z}$ element converters of various types have been constructed. ${ }^{2}, 2,3$ The converter material was usually lead, bismuth or tungsten. A common requirement for all of these converters is that they be constructed in such a manner that a suitable electric field can be applied to drift out the ionization electrons into the chamber's sensitive volume.

In this paper we report on the construction and properties of converters made of high $\mathrm{PbO}$ content fused glass tubings. In order to provide a uniform electric field within the converter for drifting out secondary electrons, the converters were treated in an elaborate hydrogen reduction process to provide a high resistivity surface layer on the inner walls of the glass tubes. The resulting resistivity was between $100-$ $1,000 \mathrm{M} \Omega / \mathrm{sq}$ and electric fields between $300-1,000$ volts $/ \mathrm{cm}$ could be readily applied without appreciable heating of the glass.

\section{Construction and Treatment of Lead Glass Converters}

The converters were constructed of the highest density lead glass that we could obtain that was sufficiently ductile so that thin wall tubings could be drawn from it. This turned out to be lead glass with a concentration of $80 \% \mathrm{PbO}$ by weight and with an overall density of $6.2 \mathrm{~g} / \mathrm{cc}$. Thin wall tubings were drawn by Garner Glass C0.4 with an ID of $1.4 \mathrm{~mm}$ and wall thickness of $0.1 \mathrm{~mm}$. The tubings were sliced to a length of $13-15 \mathrm{~cm}$ and were stacked densely into a graphite mold with a weighted top lid as shown in Figure 2. The cross-sectional area of the mold was $10 \times 10 \mathrm{~cm}^{2}$ and enough glass tubings were placed in it so that the rim of the lid had a clearance of $2 \mathrm{~mm}$. The mold and its glass tubing content were then placed in a furnace and heated until the glass softened $\left(440^{\circ} \mathrm{C}\right)$, at which point the weight of the lid compressed the tubes so that they fused together to form a solid boule. The heating and cooling cycles were done at a temperature rate of $2^{\circ} \mathrm{C} / \mathrm{min}$ and the maximum temperature was held fixed for $\frac{1}{2}$ hour to allow the tubings to fuse firmly.

The raw boule was then sawed into $2 \mathrm{~cm}$ thick slabs by a carborundum saw and polished lightly on both sides to provide smooth surfaces for the electrically conducting layers.

The surface conducting metallic lead layer was made by the hydrogen reducing treatment described by Blodgett. 5 This surface layer is more stable if, prior to the hydrogen treatment, a silicate layer is developed and hardened onto the glass surface to provide long lasting protection against moisture. This silicate layer was developed as follows. The arrays were immersed in distilled water in an uitrasonic cleaner to remove loose glass particles. They were then degreased for 5 min in acetone and again cleaned in the ultrasonic cleaner. They were then soaked in $0.1 \mathrm{M}$ $\mathrm{HCl}$ for 2 min. According to Blodgett, the acid leaches out the surface layer of $\mathrm{PbO}$ and leaves a surface silicate film 100-400 nano m thick. The arrays were again cleaned ultrasonically in distilled water to remove any remaining loose particles and the film was hardened by baking the arrays in air at $140^{\circ} \mathrm{C}$ for $8 \mathrm{hrs}$.

In the hydrogen reduction treatment as described below, a small fraction of the $\mathrm{PbO}$ is reduced to metallic lead according to the equation $\mathrm{PbO}+\mathrm{H}_{2} \rightleftharpoons \mathrm{Pb}+\mathrm{H}_{2} \mathrm{O}$. This process can take place through the protective silicate layer which is permeable to hydrogen gas. The hydrogen reduction was carried out in a vacuum tight stainless steel cylinder which was placed inside a temperature-controlled furnace as shown in Figure 3. This cylinder was $40 \mathrm{~cm}$ long so that $10-15$ converters could be treated simultaneously. In order to ensure uniformity of reduction the converters were mounted vertically with a spacing of a few $\mathrm{mm}$ between to ensure a uniform flow of the reducing gas.

Since the reduction process is reversible, very pure, dry gases were used. The stainless steel cylinder was flushed with helium and a helium atmosphere was maintained while the temperature was raised to $400^{\circ} \mathrm{C}$ at a rate of approximately $2^{\circ} \mathrm{C} / \mathrm{min}$. When $400^{\circ} \mathrm{C}$ was reached, dry, $99.99 \%$ pure $\mathrm{H}_{2}$ was admitted into the chamber at a rate of approximately $15 \mathrm{ml} / \mathrm{sec}$. This temperature and the $\mathrm{H}_{2}$ flow were maintained for $5 \mathrm{hrs}$, which is the prescribed time for optimum reduction. Then the hydrogen gas flow was replaced by helium while the oven was brought down to room temperature in about 
12 hrs. Figure 4 shows two converters, one prior to treatment and one after treatment. In order to provide a uniform electric field across the converter it is necessary to provide a uniform conducting layer to the top and bottom surfaces. For this purpose we applied a thin conductive epoxy layer by a rubber pad.

In our initial test samples we checked that the resistivity was suitably uniform along the axis of the glass tubing by use of a thin wire probe which was inserted into various holes and showed linearily within $\pm 10 \%$. We also checked the bulk resistance across the finished $10 \times 10 \times 2 \mathrm{cc}$ converters and found that the resistivity ranged from $50-100 \mathrm{M} \Omega$.

\section{Converter Efficiency Measurements}

Figure 5 shows schematically the experimental arrangement used to measure the conversion yield of electrons produced by $511 \mathrm{keV}$ gammas incident on the converter. Two detectors, consisting of a $\mathrm{NaI}$ detector and a MWPC-converter system, were placed between a positron-emitting source ${ }^{68} \mathrm{Ga}$ to detect pairs of annihilation $511 \mathrm{keV}$ gammas. The positions of the source and the NaI detector could be adjusted to accept a solid angle $\theta$ which subtends up to $\pm 45^{\circ}$ over the converter. When the size of the converter limited the solid angle to subtend less that $\pm 45^{\circ}$ we give the data both for this angle and the calculated value for $\theta= \pm 45^{\circ}$.

In the MWPC-converter system a converter was placed $4 \mathrm{~mm}$ either below or above the MWPC, which consisted of the anode plane between two orthogonal cathode planes. The anode wires (stainless steel 20 microns in diameter, spaced $3 \mathrm{~mm}$ apart) were separated by $4 \mathrm{~mm}$ from the cathode wires (stainless steel 50 microns in diameter, spaced $2 \mathrm{~mm}$ apart). The voltages applied to the MWPC and the converter to obtain maximum conversion yield are shown in Figure 5 . The MWPC and converters were enclosed in an Al box filled with a mixture of $\mathrm{Ar}(70 \%)$ and $\mathrm{CH}_{4}(40 \%)$ at $1 \mathrm{~atm}$.

The output signals from the MWPC were amplified by a charge sensitive amplifier with a conversion gain of $1 \mathrm{volt} / \mathrm{picocoulomb}$. With this gain and the voltages of the chamber as shown in Figure 5 , the $5.9 \mathrm{keV}$ line of $55 \mathrm{Fe}$ gave an output pulse of 4 volts. The discriminator threshold at which the efficiency values for the converters were measured as given in Table I was set at $100 \mathrm{mV}$, well above the noise level of the amplifier, which was $10 \mathrm{mV}$. From this we estimate that we are recording pulses produced by a minimum of 4-5 electrons. The integral curve of efficiency versus threshold voltage, Figure 6 , shows that we are extracting the electrons from the converter region with a maximum efficiency.

In Table I we give conversion efficiency values for various lead glass converters and other converters for gamma rays of $511 \mathrm{keV}$. We include a copper wire converter loaned to us by Dr. J. Votruba ${ }^{2}$ to determine the feasibility of making converters out of tungsten wires. The copper wire converter, when compared to the calculated value for the known density of wires, showed that it performs as calculated for gamma interactions with copper. We also include in the table, measured values of conversion efficiency for flat sheets of lead, tungsten and copper, which we used to check our calculations. Figure 7 shows our calculated values for lead glass converters made of tubing with $1.4 \mathrm{~mm} \mathrm{ID} \mathrm{and} 2.0 \mathrm{~cm}$ length, and for various concentrations of lead as a function of wall thickness. A hexagonal close packed array is assumed for the fused glass matrix. For the calculations we assumed an incident gamma ray angular spread of $\pm 45^{\circ}$. Comparing these calculated values with our measured values we see that there is still some discrepancy between the two in terms of drifting out all the conversion electrons.

Since one of our main interests in developing these lead glass converters was for use in a nuclear medical positron camera, as a substitute for lead on plastic converters used previously, 6 we measured the position resolution and the timing spread of the output MWPC pulse relative to the incident gamma ray.

Position resolution depends on the size and spacing of the lead glass holes. It also depends on the angle of incidence of the gamma rays, on MWPC parameters and the readout scheme. We measured the position resolution by inserting a lead collimator with an aperture of $1 \mathrm{~mm}$ between the ${ }^{68} \mathrm{Ga}$ source and the MWPC in the experimental layout of Figure 5. Figure 8 shows the spatial resolution obtained for $511 \mathrm{keV}$ gamma rays incident at approximately $90^{\circ}$ to the converter.

The spread in timing is mainly due to the drifting time differences of secondary electrons between gamma rays which interact at different depths within the converter. For a given gas mixture it depends on the electric field per unit gas pressure $E / p$ (volts/ $(\mathrm{cm} x$ $\mathrm{mm} \mathrm{Hg})$ ). Figure 9 shows our measured values for an $\mathrm{Ar}(70 \%)-\mathrm{CH}_{4}(30 \%)$ gas filling at a drift voltage of 600 volts across a $2 \mathrm{~cm}$ converter $(E / p=0.4$ volts/(cm $\times \mathrm{mm} \mathrm{Hg}))$.

\section{Conclusion}

Lead glass converters for gamma rays in the energy range $100 \mathrm{keV}$ - $10 \mathrm{MeV}$ are readily manufactured. They can be made into convenient size modules and stacked together to cover large areas. Specifically, they can be quite useful in devices that record the coincidence annihilation $511 \mathrm{keV}$ gamma rays, such as positron cameras for nuclear medicine and in solid state physics research where positron emitters are used to measure the energy distribution of the electrons within the crystals. For this latter application, the converters can be made more than a few $\mathrm{cm}$ thick, since the gamma rays are incident on the counter at angles of $90^{\circ} \pm \delta$ $\left(\delta<1^{\circ}\right)$.

\section{Acknowledgments}

We would like to thank various staff memeber of LBL who assisted us in developing this technology. Dr. R. Kopa and P. Miller designed our hydrogen treatment container and system. $D$. Anderberg and $P$.

Henrickson of the LBL glass shop were extremely helpful and we benefited greatiy from their advice and cooperation. It is also a great pleasure to thank Dr. M. Tripp of SKIA Corp. and Mr. P. P. Lin of Litton Industries who made some conductive lead glass arrays earlier, encouraging us to continue this project.

This work was supported by an N.I.H. grant to University of California, San Francisco and by the Physics Division of the $U$. S. Department of Energy under contract No. W-7405-ENG-48.

\section{References}

1) D. Chu, K. C. Tam, V. Perez-Mendez, C. B. Lim, D. Lambert, S. N. Kaplan, IEEE Trans. Nucl. Sci. $23,634(1976)$.

2) Or. Jan Votruba, private communication on copper 
wired converters; Dr. Dick Lanza from Massachusetts Institute of Technology, private communication on tungsten wired converters.

3) A. P. Jeavons, G. Charpak, R. J. Stubbs, N.I.M. 124,491 (1975).

4) Tubes were drawn by Garner Glass Co., $177 \mathrm{~S}$. Indian
Hill Rd., Claremont, CA, 97111.

5) K. B. Blodgett, Journ. of Amer. Ceram. Soc. 34,14 (1951).

6) V. Perez-Mendez, C. B. Lim, D. Ortendah1. R. Semper, A. Cheng, D. Chu, R. Hattner, L. Kaufman and D. C. Price, N.I.M. 156, 33 (1978).

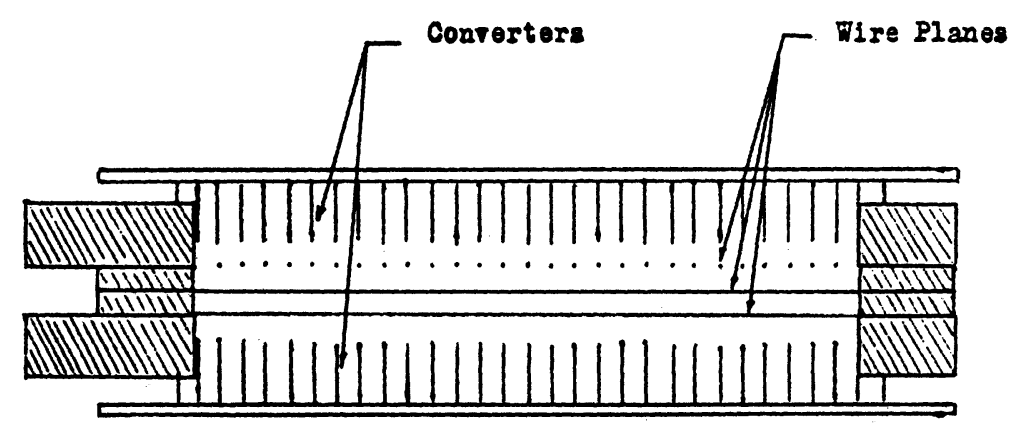

Fig. 1 Cross-sectional view of converters coupled to a conventional three wire plane MWPC. 


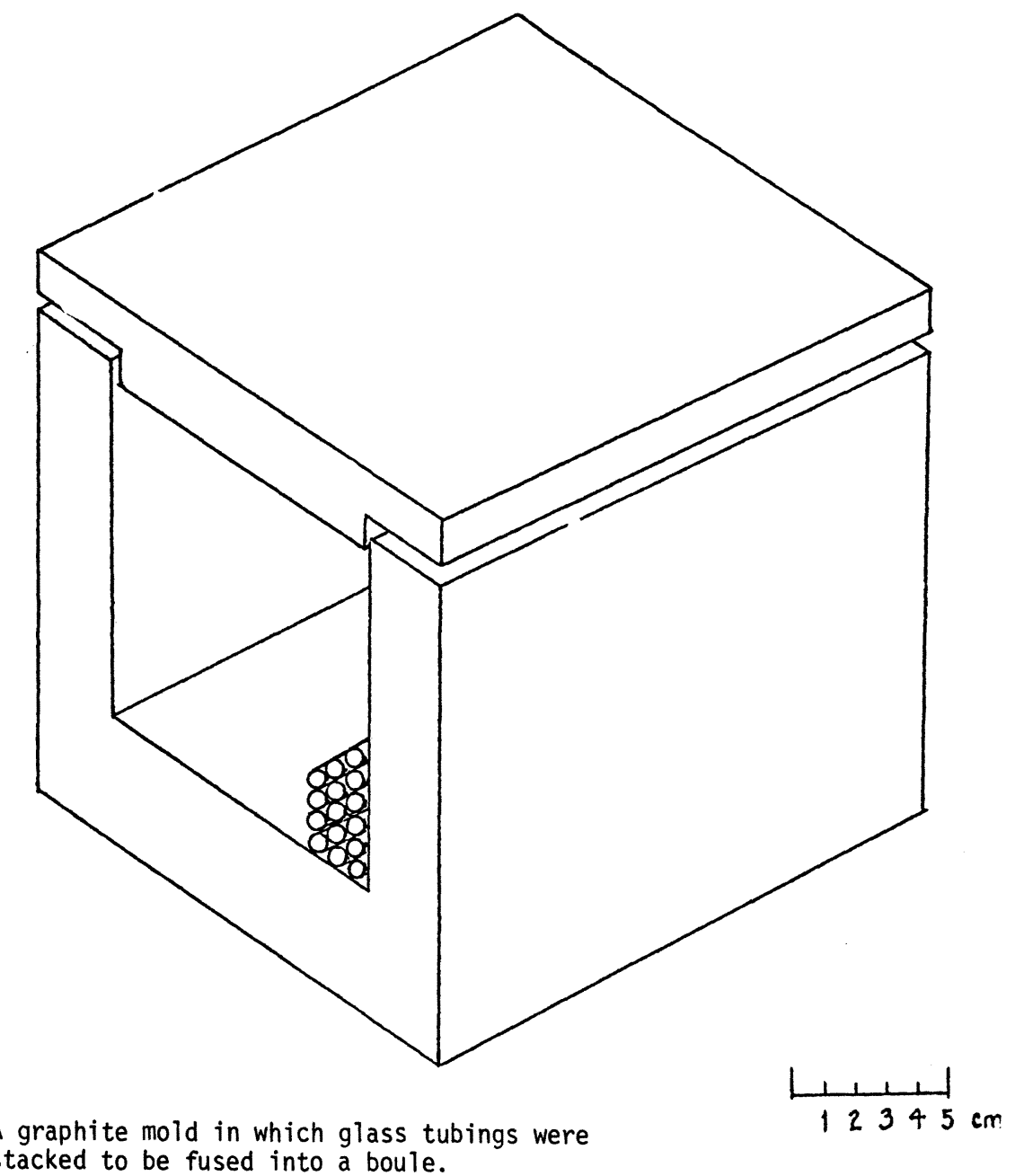

Fig. 2 A graphite mold in which glass tubings were stacked to be fused into a boule.

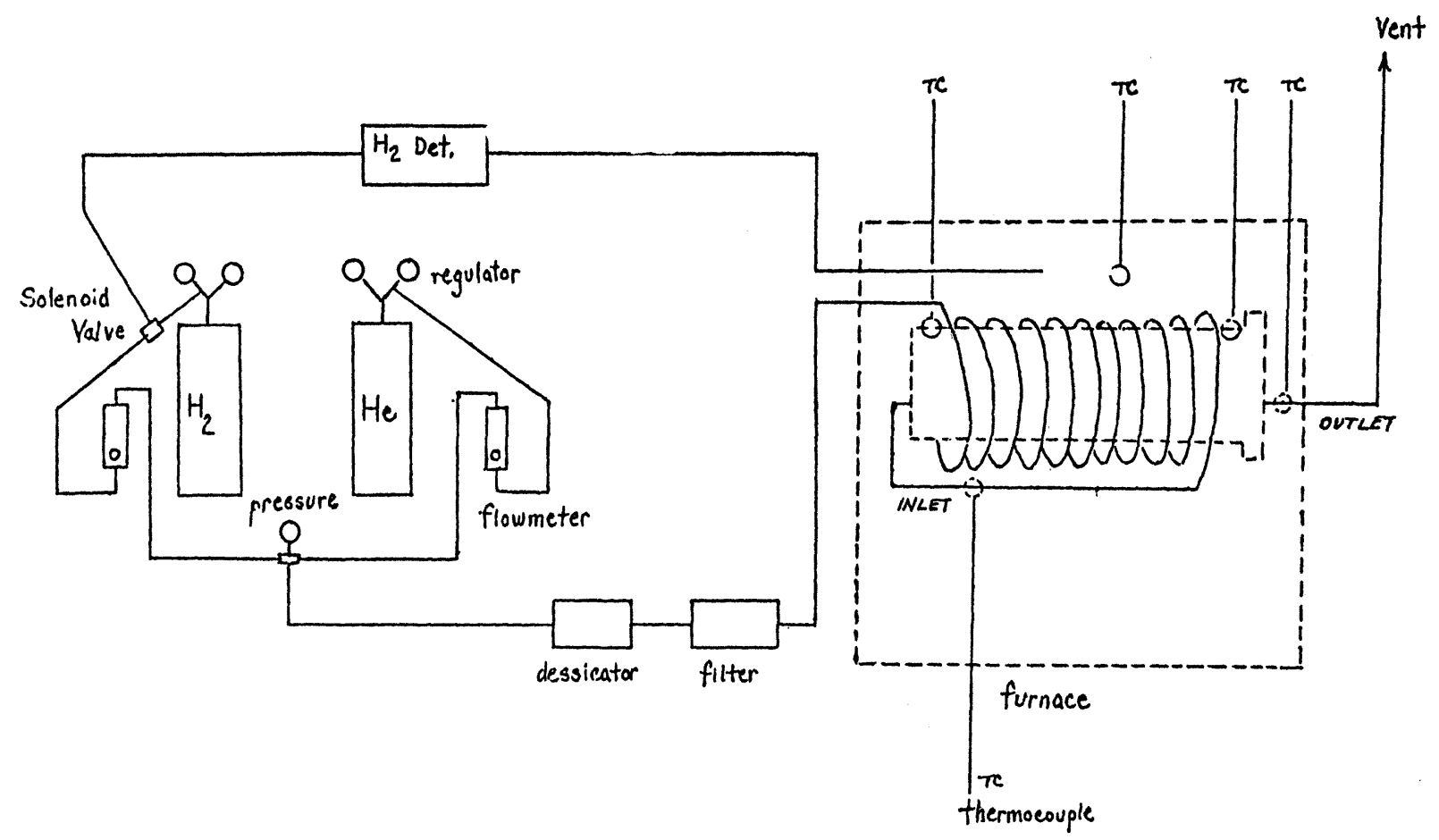

Fig. 3 The schematic diagram of the $\mathrm{H}_{2}$ treatment system. 


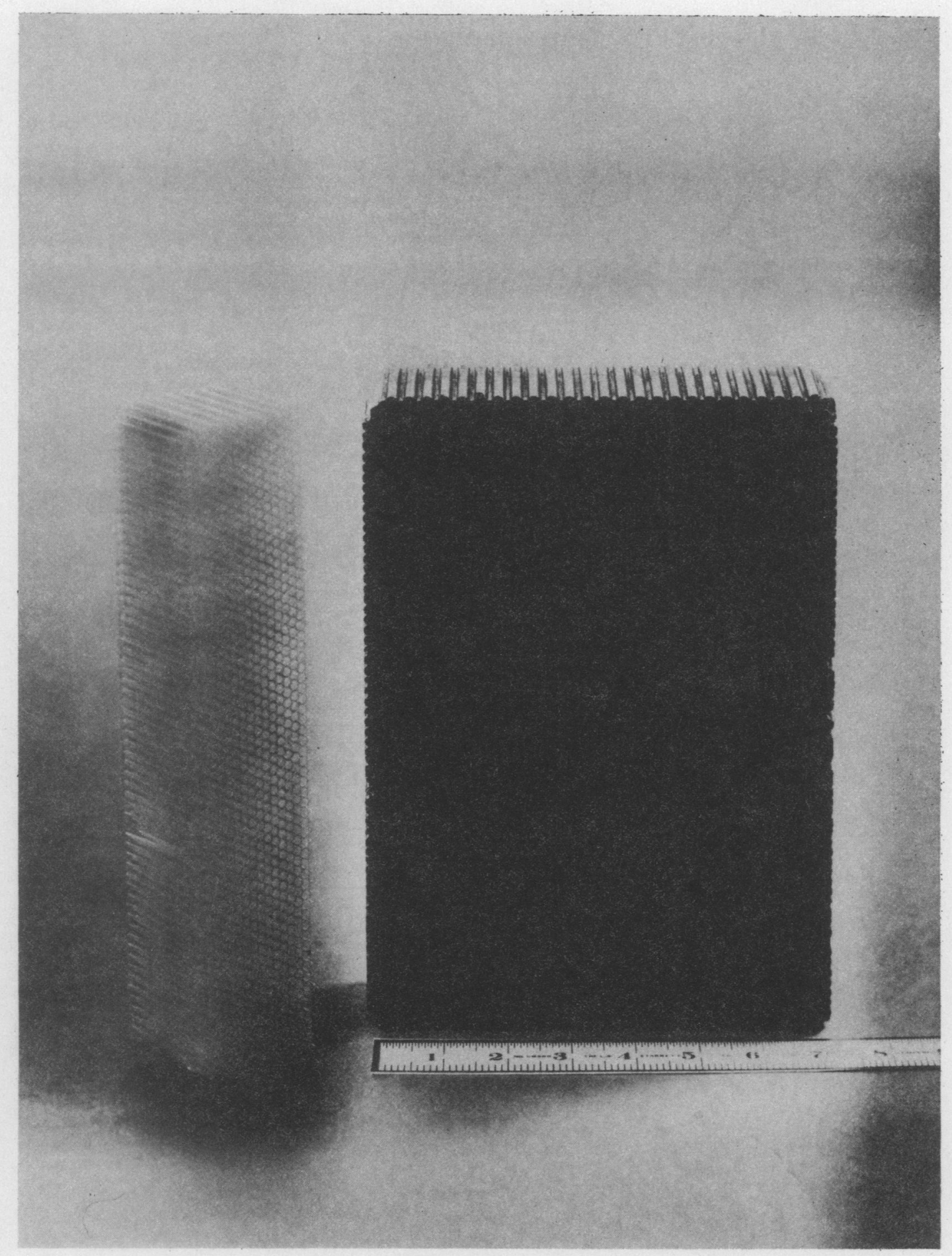

Fig. 4 The glass array on the right was treated in $\mathrm{H}_{2}$. 


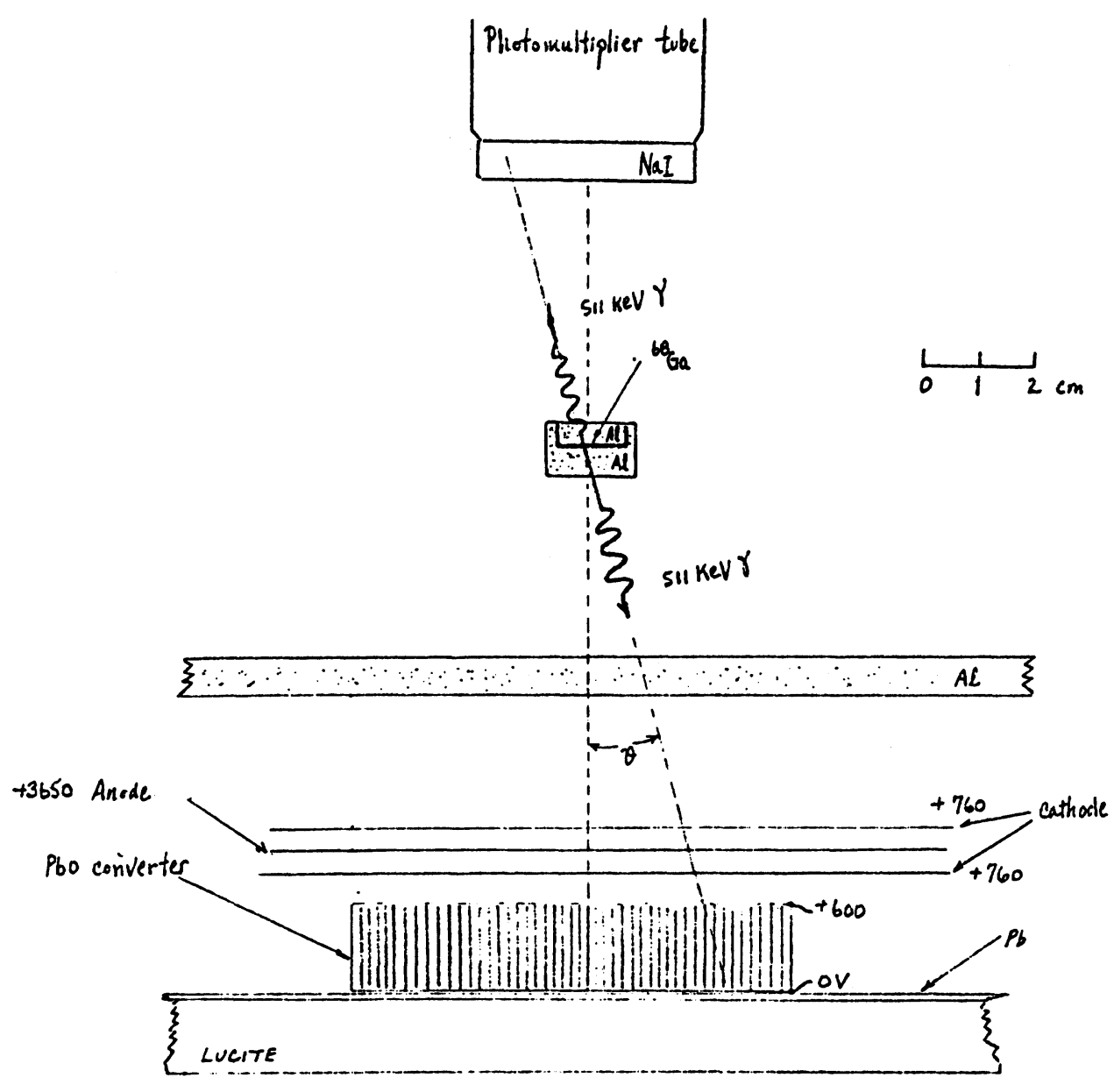

Fig. 5 Experimental arrangement used to measure the conversion yield of electrons produced by 511 $\mathrm{keV}$ gammas incident on the converter. 


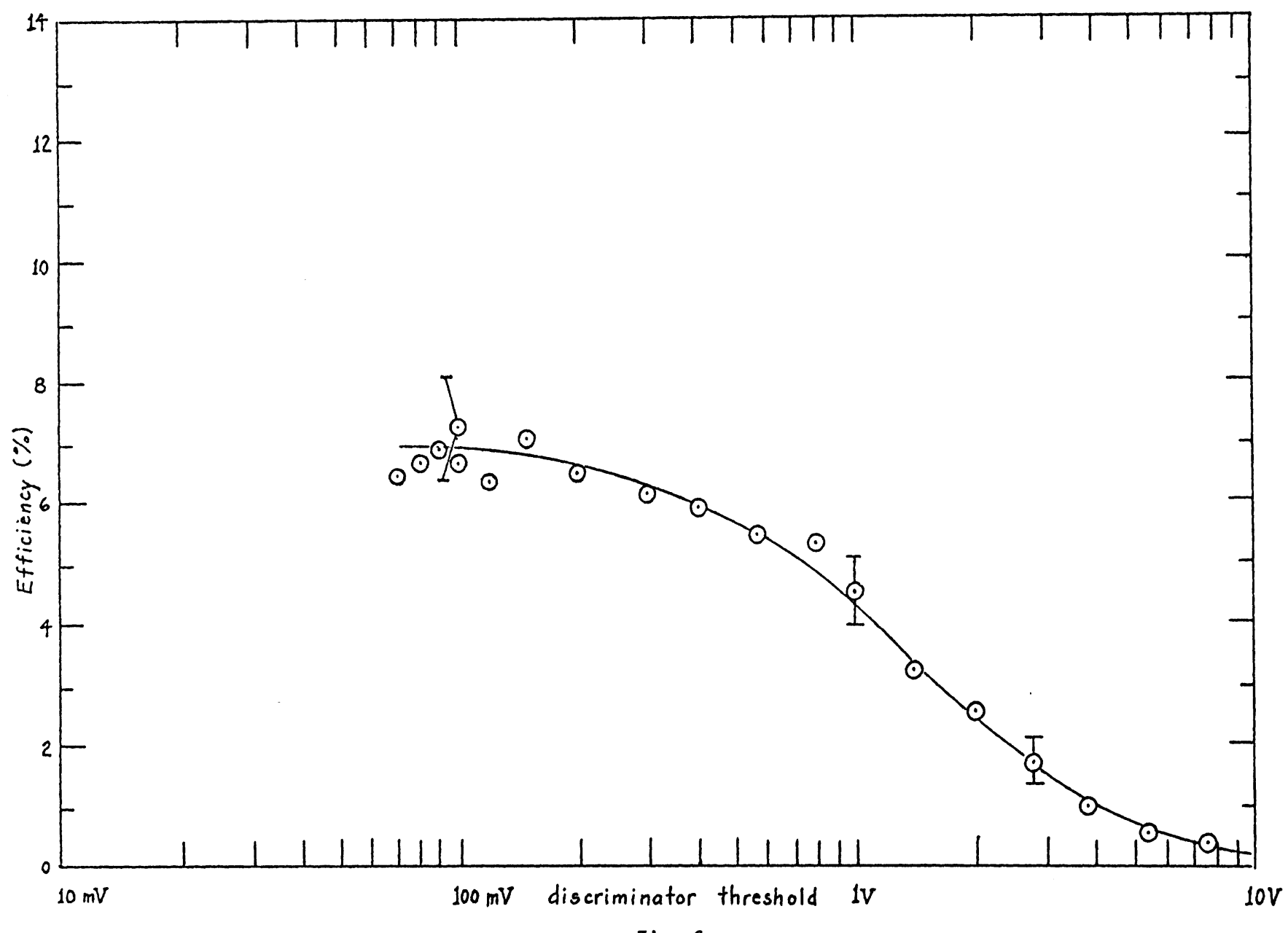

Fig. 6

Fig. 6 Electron conversion efficiency (\%) versus anode discriminator threshold voltage. 
Type of

converter
Thickness

(cm)
Maximum angle which the

solid angle subtends over the converter
Measured electron

conversion efficiency
Electron conversion efficiency for solid angle which subtends $\pm 45^{\circ}$

Cu wire converter 1.28

$53 \mathrm{Meg} \Omega 1 \mathrm{~mm}$ ID

$12^{0}$

0.0199

0.0239

$0.1 \mathrm{~mm}$ wire dia.

Cu wire converter 1.08

$12^{0}$

0.0202

0.0243

Megs Imm ID

$W$ wire converter 1.0

$12^{0}$

0.045 (estimated)

Cu sheet

0.0009

W sheet

0.0017

$\mathrm{Pb}$ sheet

0.0027

$2 \mathrm{~mm}$ inner dia.

1.5

$25^{\circ}$

0.030

0.0348

$0.43 \mathrm{~mm}$ wall $\mathrm{PbO}$

glass tubes $4.39 \mathrm{~g} / \mathrm{cc}$

$1.2 \mathrm{~mm}$ inner dia. 1.5

$0.25 \mathrm{~mm}$ wall $\mathrm{PbO}$

$25^{\circ}$

0.041

0.0476

glass tubes $4.51 \mathrm{~g} / \mathrm{cc}$

$1.00 \mathrm{~mm}$ inner dia. 1.5

$0.2 \mathrm{~mm}$ wall $\mathrm{PbO}$

$15^{0}$

0.042 at 6 1bs/in ${ }^{2} \mathrm{Ar}(70 \%)$

0.0504

glass tubes $4.04 \mathrm{~g} / \mathrm{cc}$

$1.4 \mathrm{~mm}$ inner dia. 2.0

$0.1 \mathrm{~mm}$ wall $\mathrm{PbO}$

$15^{0}$

$+\mathrm{CH}_{4}(30 \%)$

0.05 at $1 \mathrm{~atm} \mathrm{Xe}(70 \%)+0.0600$

$\mathrm{CH}_{4}(30 \%)$

0.070

0.084

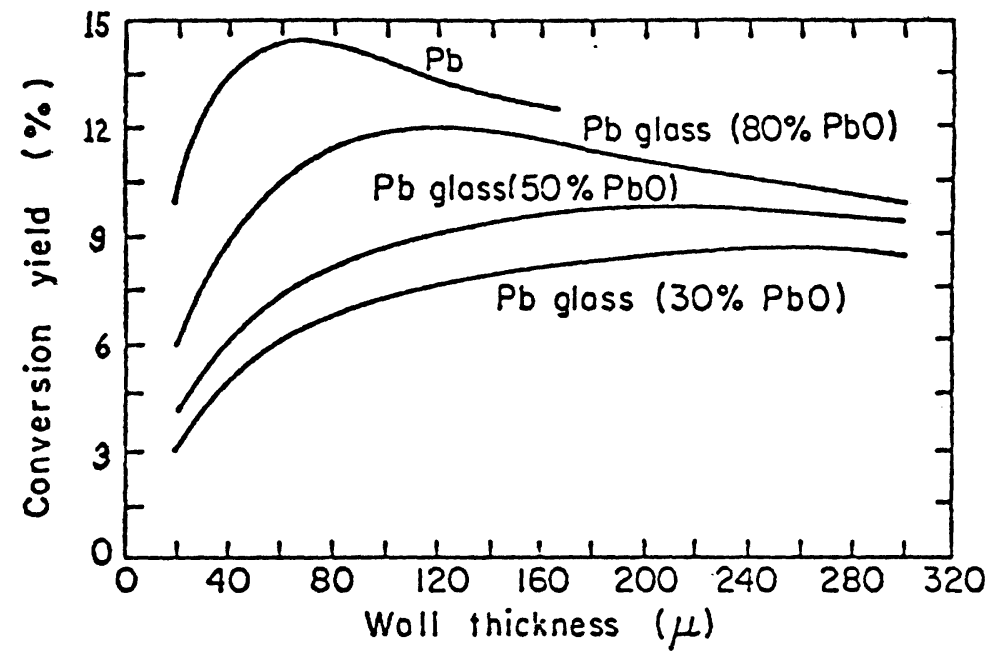

Fig. 7

Fig. 7 Calculated values for the conversion efficiency of lead glass converters $1.4 \mathrm{~mm}$ ID and 2.0 $\mathrm{cm}$ thick as a function of $\mathrm{PbO}$ concentration and wall thickness. 


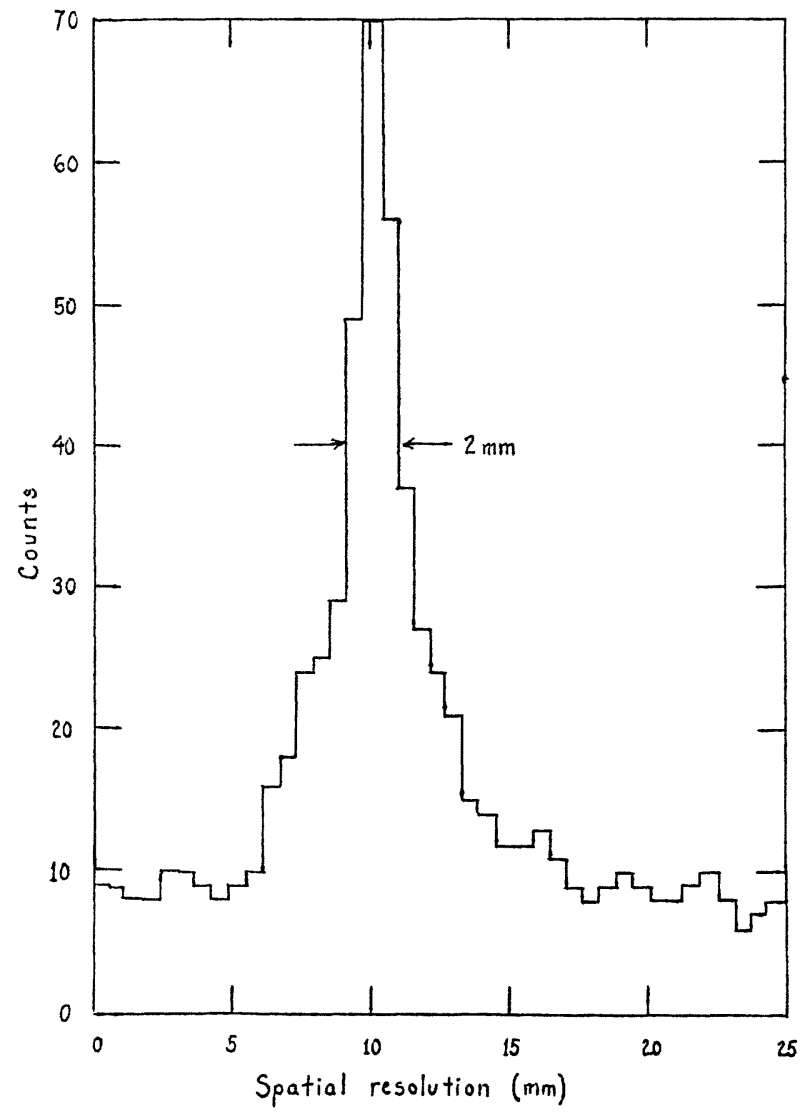

Fig. 8 Spatial resolution obtained for $511 \mathrm{keV}$ gamma rays incident at approximately $90^{\circ}$ to the converter. The gamma rays were collimated with a $1 \mathrm{~mm}$ lead slit.

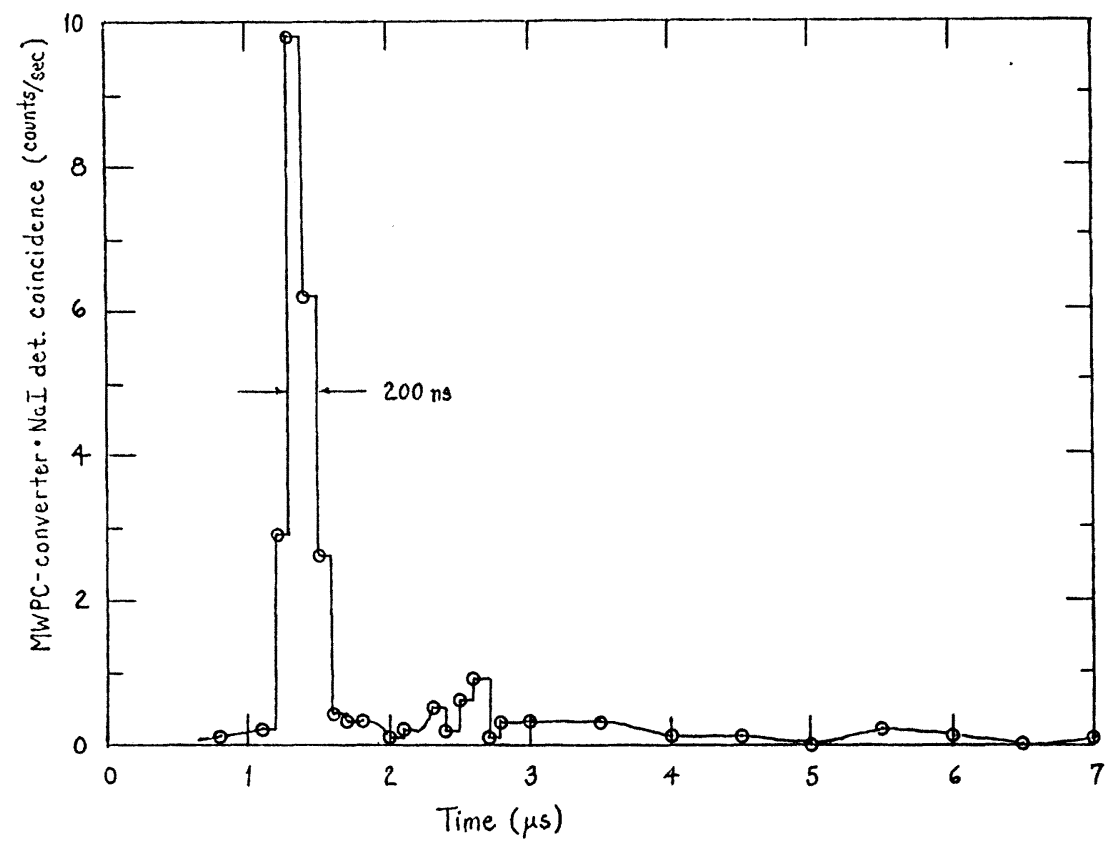

Fig. 9 Timing resolution spectrum. The width of the peak is mainly due to the drifting time differences of secondary electrons between gamma rays that interact at different depths within the converter. 